\title{
BMJ Open Effectiveness of a multicomponent safe surgery intervention on improving surgical quality in Tanzania's Lake Zone: protocol for a quasi- experimental study
}

\author{
Shehnaz Alidina (D) , ${ }^{1}$ Salome Kuchukhidze, ${ }^{1}$ Gopal Menon, ${ }^{1}$ Isabelle Citron, ${ }^{1}$ \\ Tenzing N Lama, ${ }^{1}$ John Meara, ${ }^{1,2}$ David Barash, ${ }^{3}$ Augustino Hellar, ${ }^{4}$ \\ Ntuli A Kapologwe, ${ }^{5}$ Erastus Maina, ${ }^{6}$ Cheri Reynolds, ${ }^{7}$ Steven J Staffa, ${ }^{8}$ \\ Alena Troxel, ${ }^{9}$ Asha Varghese, ${ }^{3}$ David Zurakowski, ${ }^{8}$ Mpoki Ulisubisya, ${ }^{10}$ \\ Sarah Maongezi ${ }^{11}$
}

To cite: Alidina S, Kuchukhidze S, Menon G, et al. Effectiveness of a multicomponent safe surgery intervention on improving surgical quality in Tanzania's Lake Zone: protocol for a quasiexperimental study. BMJ Open 2019;9:e031800. doi:10.1136/ bmjopen-2019-031800

- Prepublication history and additional material for this paper are available online. To view please visit the journal (http:// dx.doi.org/10.1136/bmjopen2019-031800).

SA and SK are joint first authors. MU and SM are joint senior authors.

Received 19 May 2019 Revised 07 August 2019 Accepted 12 September 2019

D) Check for updates

(C) Author(s) (or their employer(s)) 2019. Re-use permitted under CC BY-NC. No commercial re-use. See rights and permissions. Published by BMJ.

For numbered affiliations see end of article.

Correspondence to Dr Shehnaz Alidina;

Shehnaz_Alidina@hms.harvard. edu

\section{ABSTRACT}

Introduction Effective, scalable strategies for improving surgical quality are urgently needed in low-income and middle-income countries; however, there is a dearth of evidence about what strategies are most effective. This study aims to evaluate the effectiveness of Safe Surgery 2020, a multicomponent intervention focused on strengthening five areas: leadership and teamwork, safe surgical and anaesthesia practices, sterilisation, data quality and infrastructure to improve surgical quality in Tanzania. We hypothesise that Safe Surgery 2020 will (1) increase adherence to surgical quality processes around safety, teamwork and communication and data quality in the short term and (2) reduce complications from surgical site infections, postoperative sepsis and maternal sepsis in the medium term.

Methods and analysis Our design is a prospective, longitudinal, quasi-experimental study with 10 intervention and 10 control facilities in Tanzania's Lake Zone. Participants will be surgical providers, surgical patients and postnatal inpatients at study facilities. Trained Tanzanian medical data collectors will collect data over a 3-month preintervention and postintervention period. Adherence to safety as well as teamwork and communication processes will be measured through direct observation in the operating room. Surgical site infections, postoperative sepsis and maternal sepsis will be identified prospectively through daily surveillance and completeness of their patient files, retrospectively, through the chart review. We will use difference-in-differences to analyse the impact of the Safe Surgery 2020 intervention on surgical quality processes and complications. We will use interviews with leadership and surgical team members in intervention facilities to illuminate the factors that facilitate higher performance.

Ethics and dissemination The study has received ethical approval from Harvard Medical School and Tanzania's National Institute for Medical Research. We will report results in peer-reviewed publications and conference presentations. If effective, the Safe Surgery 2020

\section{Strengths and limitations of this study}

- A longitudinal, controlled, quasi-experimental, multisite design will ensure an adequately powered, replicable and internally/externally valid study that offers high-quality evidence on the effectiveness of the Safe Surgery 2020 intervention in improving surgical quality processes and reducing surgical complications.

- Qualitative methods will illuminate the factors that allow facilities to achieve high performance and will contribute to knowledge for effective scale-up.

- The use of prospective and direct observation methods, trained Tanzanian medical data collectors familiar with the local context, and weekly data quality checks will contribute to the collection of high-quality data.

- There is potential for under-reporting of surgical site infections and postoperative sepsis since the study will not follow patients after discharge.

- There is a possibility that reducing surgical complications may take beyond the 9-month intervention period; further longitudinal data collection may be necessary to understand the full impact of the Safe Surgery 2020 intervention.

intervention could be a promising approach to improve surgical quality in Tanzania's Lake Zone region and other similar contexts.

\section{INTRODUCTION}

The recognition of surgery as a critical component of universal health coverage has led to a worldwide movement to expand access to surgery in low-income and middle-income countries (LMICs). In 2015, the Lancet Commission on Global Surgery reported that 


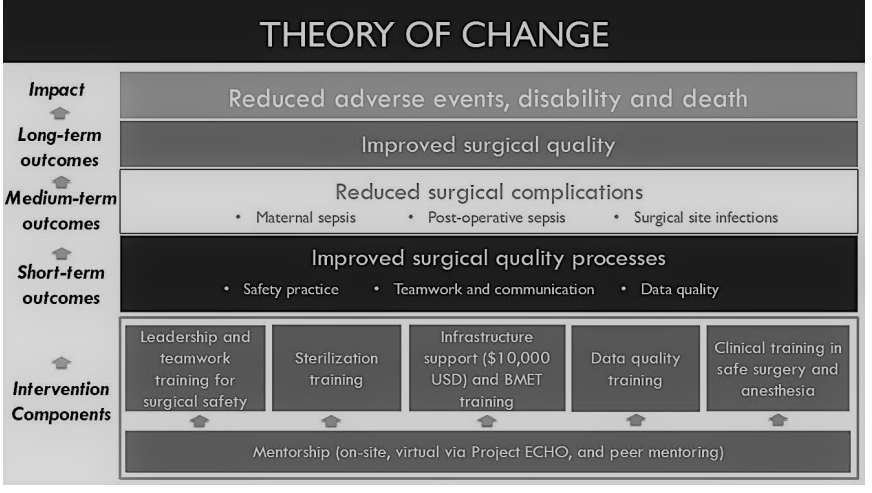

Figure 1 Safe surgery 2020 theory of change. BMET, Biomedical Engineer Technician; ECHO, Project Extention for Community Healthcare Outcomes

about one-third of all deaths globally were from conditions requiring surgery, and 77.2 million disability-adjusted life-years could be prevented annually by basic surgical treatment. ${ }^{1}$ However, while surgery can offer positive health outcomes, it also causes preventable complications and deaths globally, with LMICs experiencing a disproportionate share. ${ }^{12}$ As health systems improve access to surgical services in LMICs, it is important to ensure that surgery is safe and preventable complications and deaths are averted.

Despite its importance, there is a dearth of evidence on how best to improve surgical quality in LMICs. ${ }^{34}$ While a recent systematic review found 350 studies examining surgical quality in LMICs, the overall quality of evidence was weak. ${ }^{3}$ About $80 \%$ of the studies were observational and about $80 \%$ focused only on a single centre, most often a regional centre. Basic parameters, such as facility type or follow-up period, were not always reported, and few studies fully described the intervention or context. ${ }^{3}$ Experts on healthcare quality in LMICs suggest that single-component interventions have had a modest effect on performance; they suggest that multicomponent interventions may be more effective and further research is needed on these approaches. ${ }^{4-9}$

Safe Surgery 2020 in Tanzania, a collaboration between Assist International, Dalberg Advisors, Jhpiego and Harvard's Program in Global Surgery and Social Change, offers one approach to improving surgical quality. Safe Surgery 2020's approach includes partnering with the Tanzanian government; consulting front-line leadership and surgical teams to identify priorities; a multicomponent intervention to improving surgical quality; and evaluating the intervention to examine how best to improve surgical quality in LMICs.

Safe Surgery 2020's intervention addresses key surgical quality issues in Tanzania. Studies have found poor adherence to quality and safety practices and poor patient outcomes following surgery in Tanzania. Evidence-based safety, teamwork and communication practices known to improve surgical quality in high-resource settings, such as WHO's Surgical Safety Checklist, are not consistently used. ${ }^{10}$ Checklist use is associated with reduced morbidity, ${ }^{211-14}$ reduced mortality, ${ }^{211} 14$ improved safety attitudes, ${ }^{15} 16$ and improved teamwork and communication. ${ }^{12} 1718$ Studies in Tanzania have found deficits in anaesthesia practices, ${ }^{19}$ inconsistent administration of prophylactic antibiotics for $\mathrm{C}$-section patients and missing information in patient records. ${ }^{20}{ }^{21}$ In terms of patient outcomes, studies in regional centres have found surgical site infection (SSI) rates in Tanzanian patients ranging from $10.9 \%$ to $19.4 \%,{ }^{22}{ }^{23}$ comparable to rates reported for other LMICs but higher than rates of high-income countries. ${ }^{24-26}$ The disparity for women who undergo C-sections is even more significant. SSI rates among Tanzanian C-section patients in tertiary hospitals are reported at 48\%, 5-18 times higher than rates in high-income countries. ${ }^{27-29}$ Streamlining surgical care processes to implement the best practices for infection prevention offers promise in reducing SSI rates and improving surgical quality. ${ }^{30}$

Figure 1 shows Safe Surgery 2020's theory of change for improving surgical quality in Tanzania. Programme partners will implement a multicomponent intervention to strengthen five crosscutting surgical quality areas: leadership and teamwork, safe surgical and anaesthesia practices, sterilisation, data quality and infrastructure. The aim is to improve surgical quality processes related to safety, teamwork and communication, and data quality that in turn will reduce surgical complications related to SSIs, postoperative sepsis and maternal sepsis. Ultimately, improved surgical quality will result in fewer complications from the surgical care.

This paper describes the methods to evaluate the impact of the Safe Surgery 2020 intervention in improving surgical quality in 20 health facilities in the Lake Zone of Tanzania. Our study will address two hypotheses: (1) in the short term, the intervention will improve adherence to surgical quality processes related to safety, teamwork and communication, and data quality, and (2) in the medium term, the intervention will reduce complications from SSIs, postoperative sepsis and maternal sepsis.

\section{METHODS AND ANALYSIS \\ Study design}

The study design includes two main elements: (1) a longitudinal, controlled quasi-experimental study examining changes in surgical quality processes as well as postoperative and postnatal complications; and (2) an in-depth, longitudinal qualitative study exploring factors that distinguish high-performing facilities with the most improvement in surgical quality processes. The study will have 3-month preassessment, 9-month intervention and 3-month postassessment periods (figure 2).

The quantitative results will illuminate the impact of the intervention, while studying 'positive deviants' with the most improvement in adherence to surgical quality processes measured by safety as well as teamwork and communication scores using qualitative methods will identify the factors that allow facilities to achieve top 


\section{Baseline quantitative data} collection (3 months)

\section{-Baseline qualitative} interviews

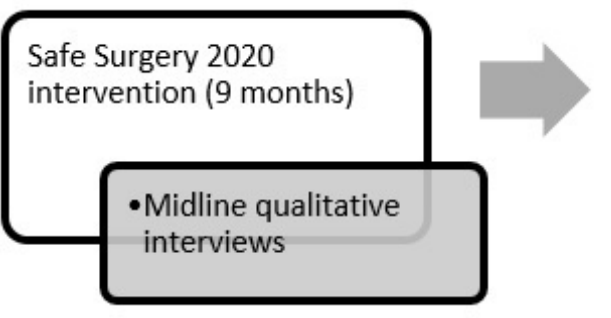

Endline quantitative data collection (3 months)

- Endline qualitative interviews
Figure 2 Project timelines. performance. Combined, the results will contribute to knowledge about the effectiveness of strategies to improve similar contexts.

Safe Surgery 2020 intervention will be introduced at 10 intervention facilities, while 10 control facilities will not receive the intervention. The intervention will be delivered at the facility level, with the primary outcome measured at the individual level (ie, surgical provider or patient).

\section{Setting and site selection} Victoria, Tanzania: Geita, Simiyu, Shinyanga, Kagera and Mara. Collectively, they have an estimated population of 9060348 (18.5\% of the nation's population); $28.2 \%$ live below the poverty line and $67.0 \%$ live in rural areas. ${ }^{31}$

Ten facilities within Mara and Kagera regions have been selected as intervention sites based on a feasibility surgical quality in Tanzania's Lake Zone region and other

The setting includes five regions surrounding Lake assessment conducted by Safe Surgery 2020 partners in October 2017 using a priori selection criteria: regional, district or health centre facilities with a surgical volume of at least 50 major surgeries annually; perceived quality improvement (QI) culture; availability of basic infrastructure to support the implementation of QI processes; willingness of facility leadership and surgical teams to participate; and site accessibility for the research team. Ten sites in Geita, Shinyanga and Simiyu have been selected as control sites based on their similarity to the intervention sites on community socioeconomic characteristics, facility level, type of surgical cases and total surgical volume. Facility characteristics are outlined in table 1 .

Results of the feasibility assessment found that there are seven surgeons and no anaesthesiologists across the study facilities, surgery is largely provided by generalist physicians and assistant medical officers with 5 years of clinical
Table 1 Characteristics of intervention and control facilities, 2018
Characteristics

All facilities

$(\mathrm{n}=20)$

n (\%)

Level of facility

Health centre

District hospitals

Regional referral hospital

No of inpatient beds

0-100

101-300

$300+$

Average monthly surgical procedures per facility

Average monthly bellwether procedures per facility

\begin{tabular}{|c|c|c|c|}
\hline Caesarean sections & 44 & 46 & 42 \\
\hline Laparotomies & 6 & 5 & 8 \\
\hline Open-fracture fixations & 0 & 0 & 0 \\
\hline Average monthly inpatient volume per facility & 589 & 330 & 258 \\
\hline \multicolumn{4}{|c|}{ Surgical, obstetric and anaesthesia specialists for regions } \\
\hline Certified obstetrics/gynaecologists & 2 & 1 & 1 \\
\hline Anaesthesiologists & 0 & 0 & 0 \\
\hline
\end{tabular}

$(n=10)$

n (\%)

$\begin{array}{ll}(n=10) & (n=10) \\ n(\%) & n(\%)\end{array}$

$\begin{array}{lll}4(20) & 2(20) & 2(20) \\ 11(55) & 6(60) & 5(50) \\ 5(25) & 2(20) & 3(30)\end{array}$

$5(25)$

3 (30)

2 (20)

$13(65)$

$6(60)$

7 (70)

2 (10)

1 (10)

1 (10)

90

75

Control facilities 


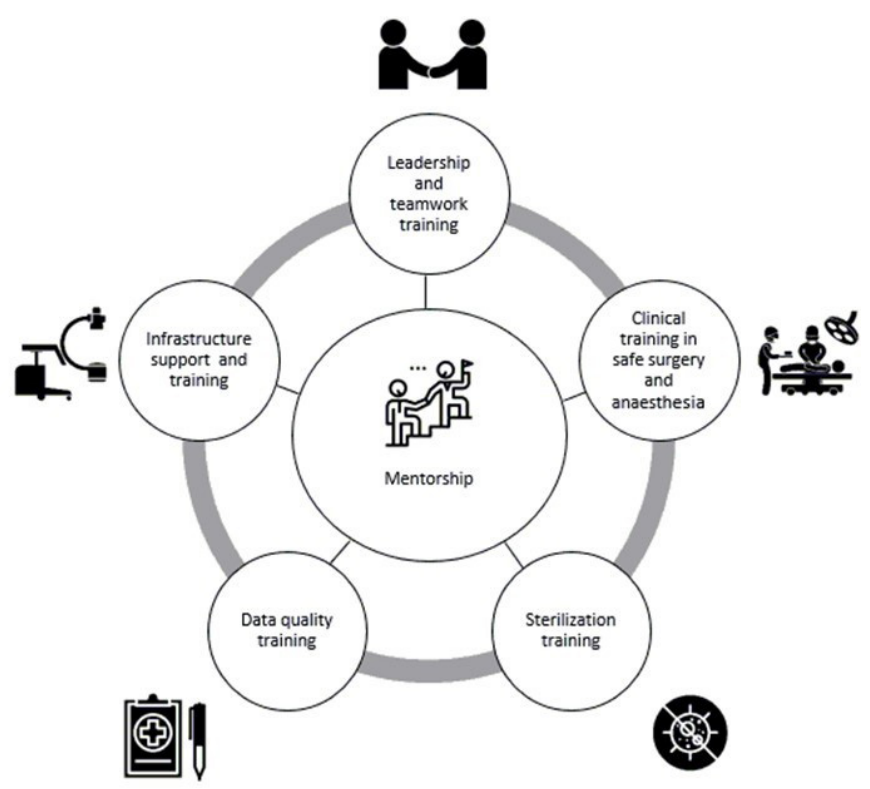

Figure 3 Safe surgery interventions.

training and anaesthesia is provided by nurse anaesthetists. Infrastructure, such as blood, clean water, oxygen and electricity, is not consistently available. Only two of the three Bellwether procedures, caesarean sections and laparotomies, are performed, with no open fracture fixations performed. Most facilities lack postoperative recovery areas and intensive care units. The Surgical Safety Checklist is not in use at any facility.

\section{Study population}

All surgical inpatients who had a major surgery and postnatal women (based on WHO consensus definition) who underwent C-sections or delivered vaginally ${ }^{32}$ at study facilities will be enrolled and followed during their inpatient stay, up to 30 days. All patients who have a surgical procedure in the major operating theatre are operationally defined as having a major surgery whether performed under local or general anaesthesia. Patients from the study population will not be followed after discharge. Paediatric patients under the age of 5 years, minor surgeries, visiting surgeons' patients, antenatal patients, women with spontaneous abortions and surgical outpatients will be excluded. Excluded minor surgeries are provided in online supplementary appendix 1 .

\section{Patient and public involvement}

Patients were not involved in the conception and design of this study.

\section{Intervention}

Surgery 2020 is a multicomponent intervention that acts across the surgical system to strengthen five surgical quality areas: leadership and teamwork, safe surgery and anaesthesia practices, sterilisation, data quality and infrastructure, with staggered implementation over 9 months. The intervention will be delivered through a series of training sessions attended by a multidisciplinary surgical team from each intervention facility.

During the leadership and teamwork training, surgical teams will identify surgical QI priorities by considering impact, influence and ability; identify root causes using QI techniques such as the fishbone diagrams or "Five Whys' to solve problems and develop a QI plan to implement at their facility over 9 months. ${ }^{33} 34$

Accurate recording and reporting of outcome measures is key for feedback on performance, thus encouraging surgical QI and patient safety. ${ }^{35}$ The data quality strengthening intervention will improve the data accuracy and enable mentors to form feedback loops with surgical teams to identify gaps in quality.

Intervention facilities will also develop a Facility Accelerator Fund grant proposal to access a US\$10000 grant to address infrastructural barriers to implementing their QI plan.

A mentoring team including a surgeon, anaesthesiologist, obstetrician and a theatre nurse from the zonal hospital will visit each intervention facility bimonthly to reinforce the training and to support the surgical teams in achieving their QI plan. Mentoring will also occur virtually through Project ECHO (Project Extension for Community Healthcare Outcomes), ${ }^{36}$ where specialists from the zonal hospital and international faculty will provide didactic clinical updates, demonstrate skills and techniques, and provide weekly advice on difficult cases using video conferencing. Intervention facilities will learn from each other's experiences through a WhatsApp group and an annual knowledge sharing session.

To promote sustainability, facilitating leadership support, buy-in at all levels, and a QI culture and structure will be emphasised. ${ }^{30}$ Safe Surgery partners will offer structured feedback through facility-level baseline results and data on performance and coaching through mentorship visits. Safe Surgery 2020's collaboration with the ministry to develop the National Surgical Obstetric and Anaesthesia Plan further explores sustainable financing mechanisms for QI at scale. ${ }^{37}$

The intervention is shown in figure 3; additional information is provided in online supplementary appendix 2 .

\section{Outcomes}

Short-term outcomes include surgical quality processes related to safety, teamwork and communication, and data quality. Medium-term outcomes are rates of SSIs, postoperative sepsis and maternal sepsis, up to a 30-day postoperative or postnatal hospitalisation period. The outcomes are described in box 1 .

\section{Data collection and analysis}

Quantitative data collection and analysis

Twenty-five Tanzanian medical doctors will be recruited as data collectors and trained by the research team for 4 days in the identification and classification of surgical process measures and complications, study data collection tools and Research Electronic Data Capture (REDCap). ${ }^{38}$ Data collectors will be placed at each of the 20 study sites during 


\section{Box 1 Short-term and medium-term study outcomes}

\section{Short-term outcomes}

1. Patient safety process adherence rate: Rate of six indicators: use of pulse oximetry at the time of initiation of anaesthesia; administration of prophylactic antibiotics within 60 min before incision; oral confirmation, immediately before incision, of the identity of the patient, operative site and procedure to be performed; completion of an instrument, sponge and needle count at the end of the procedure; appropriate operative site cleansing (skin preparation with Chlorhexidine-alcohol or iodine-based solution); and vaginal preparation with povidone-iodine. ${ }^{213346465}$

2. Teamwork and communication discussion adherence rate: Rate of eight discussion items: risk for airway difficulty/aspiration; risk of blood loss; surgical provider and anaesthetist's discussion on patient-specific concerns; sterility of instruments and equipment; anticipated procedure level of difficulty and duration; equipment/ instrument problems that arose during surgery and concerns related to patient's postoperative recovery. ${ }^{21718}$

3. Patient medical record completion rate: Rate of documentation of vitals and of surgical site infection (SSI) or sepsis and their symptoms in patient files; perioperative monitoring (postoperative notes, indication for C-section) and completeness of inpatient clinical progress notes (patient history, progress notes, daily doctor's orders and notes). ${ }^{66} 67$

\section{Medium-term outcomes}

4. SSI rate: Rate of SSIs (superficial, deep or organ/space) diagnosed within 30 days of surgery or until discharge, among all current or returning postoperative inpatients.

5. Postoperative sepsis rate: Rate of postoperative sepsis diagnosed within 30 days of surgery or until discharge, among all current or returning postoperative inpatients.

6. Maternal sepsis rate: Rate of maternal sepsis diagnosed within 30 days of delivery (vaginal or through $\mathrm{C}$-section) or until discharge, among all current or returning obstetric inpatients.

the 3-month preintervention and 3-month postintervention period. Data collectors will work full time and will be compensated. They will collect data prospectively on each operation and birth using three standardised data collection tools. Data will be collected first using paperbased tools and transferred electronically into REDCap daily. Data collection tools are provided in online supplementary appendix 3 .

\section{Surgical safety checklist observation tool}

Data collectors will observe each operation and record the surgical teams' adherence to essential surgical safety as well as teamwork and communication quality processes using the Surgical Safety Checklist Observation Tool adapted from Huang et al. ${ }^{39}$ The tool contains 38 yes/no or not applicable items in the following categories: sign-in (13 items), time out (14 items), sign out (6 items) and additional items (5 items). Case information, including demographics, clinical and procedural information, will be entered for each patient from the medical record.
Daily surveillance log and SSI, postoperative sepsis and maternal sepsis screening tools

Data collectors will follow patients prospectively during their in-patient stay, up to 30 days, for SSI, postoperative sepsis and maternal sepsis. Patient demographics will be collected and outcomes will be identified based on clinical symptoms through daily surveillance during patient rounds, communication with clinical staff and patient chart review. If symptoms are identified, additional screening tools will be administered to collect information on patient demographics, medical history, symptoms and severity of infection and antibiotic treatment. The SSI Tool was adapted from the Protocol for the Surveillance of Surgical Site Infection, Public Health England (2013) and based on the CDC criteria for diagnosing and classifying SSIs. ${ }^{26} 40$ The Postoperative Sepsis Screening Tool and Maternal Sepsis Tool were adapted for low-resource settings from the Surviving Sepsis Campaign guidelines, which are based on the Second (2001) International Consensus on Sepsis. ${ }^{41-44}$ The definitions for diagnosing and classifying SSIs, postoperative and maternal sepsis are provided in online supplementary appendix 4 .

\section{Data quality assessment tool}

Paper-based data quality will be assessed retrospectively in the intervention regions only; medical records of all patients diagnosed with SSI, sepsis and maternal sepsis will be reviewed before and after the intervention. The questionnaire includes 18 yes/no questions related to documentation of vital signs, SSIs/sepsis symptoms, patient history, daily progress notes, doctors' orders, partogram utilisation, discharge details and postoperative notes. The tool was informed by WHO Western Pacific Region's Medical Records Manual: A Guide for Developing Countries ${ }^{45}$ and measures change in the completeness of medical records for patients diagnosed with SSIs, postoperative sepsis and maternal sepsis.

\section{Data quality assurance}

A data quality assurance system will focus on four data quality components: accuracy (correct data values), completeness (no missing data elements), reliability (data collected consistently across study sites) and timeliness (data recorded and reported the same day, on a near real-time basis). Our processes for achieving high-quality data include training data collectors, a standardised operating procedure manual, an electronic data capture with built-in quality controls and weekly data quality checks at each study site by the field research team.

\section{Power analysis}

Based on the feasibility assessment results, we anticipate an average monthly surgical volume per site of 75 cases in intervention regions and 90 cases in control regions. Over the 3-month baseline period, we assume that this will result in at least 2250 enrolled patients in intervention and control regions, respectively. The sample size was calculated to provide $80 \%$ power based on the 
Cochran-Armitage test for detecting a $5 \%$ reduction in the rate of SSIs, a $3 \%$ reduction in the sepsis rate and a $1.5 \%$ reduction in the maternal sepsis rate postintervention. These sample sizes will provide $80 \%$ statistical power to detect a difference in average Surgical Safety Checklist adherence of $5 \%$ between the control and intervention arms using Student's t-test, assuming an SD in adherence of 20\% (standardised effect size $=0.25$ ). All statistical tests assume a two-tailed alpha level of 0.05 . Power analysis was performed using nQuery Advisor software (V.8.0, Statistical Solutions, Cork, Ireland).

\section{Data analysis}

To evaluate the impact of the intervention and compare changes in outcome rates over time, we will conduct difference-in-differences analysis. The slope fitted in the mixed-effects generalised linear model will be used to evaluate the difference-in-differences of SSI, sepsis, maternal sepsis and Surgical Safety Checklist adherence rates. For the analysis of SSI, postoperative sepsis and maternal sepsis, we will assume a binomial distribution while considering region, facility and patient as random effects. Both C-sections and vaginal deliveries will be included in the maternal sepsis rate calculation although stratified analysis by mode of delivery will be performed. Given that the power calculation is performed based on surgical cases alone, we are unlikely to be powered to detect a change in maternal sepsis rates only among women who undergo C-sections. For the analysis of Surgical Safety Checklist adherence, we will consider a normal distribution with region, facility and patient as random effects. The association between Surgical Safety Checklist adherence and SSI, postoperative sepsis and maternal sepsis will be evaluated by treating Surgical Safety Checklist adherence as a categorical variable. Collapsed categories for Surgical Safety Checklist adherence will be defined based on tertile (low, moderate and high adherence) categories. Covariates in all models will include intervention region, age, sex, procedure type, wound class, ASA score and length of surgical procedure. Adjusted ORs, 95\% CIs and $\mathrm{p}$ values based on the Wald test will be presented to estimate the independent association of each factor with SSI, sepsis, maternal sepsis and adherence to the Surgical Safety Checklist. In secondary analyses, outcome measures will be stratified by health centre, district health facility and regional health centre to estimate differences in change rates by facility level, controlling for facility-level influences on outcomes. All statistical analyses will be performed using Stata software (V.15.0, StataCorp). A two-tailed significance level of $\mathrm{p}<0.05$ will be used as the criteria for statistical significance.

\section{Qualitative data collection and analysis}

\section{Identification of higher and lower performing intervention facilities}

We will use a positive deviance approach with information-rich cases to understand the factors that distinguish facilities with the most surgical quality improvement. ${ }^{4647} \mathrm{To}$ maximise our learning, we will use a purposeful sample of six facilities as polar examples. We will stratify our sample of 10 facilities into higher and lower performing facilities based on quantitative findings. Higher performers will be defined as the three facilities with the most improvement in surgical quality processes measured by safety as well as teamwork and communication scores (change in percentage points). Lower performers will be defined as the three facilities with the least improvement in surgical quality processes.

\section{Distinctions between higher and lower performing groups}

We will analyse differences between higher and lower performing groups using interview data collected at baseline, midline and conclusion. The interviews will explore contextual factors, implementation processes, facilitators and barriers to implementation as well as their acceptability. Interviewees will include a facility leader and three members of the surgical team at each site. Semistructured interviews will be conducted using a standard interview guide, digitally recorded based on participant consent, transcribed and entered into NVivo software (V.12; QSR International) for coding.

We will use the constant comparison method to analyse the qualitative data between higher and lower performing facilities. ${ }^{46}{ }^{48}$ First, study team members will review and discuss responses to assign preliminary codes to represent recurring themes. We will establish intercoder reliability by having two independent researchers code and compare results for an initial set of transcripts, with any disagreements resolved through discussion. Codes will be refined to represent the themes reflected in the data. We will reanalyse the transcripts using the final coding scheme and then rate the strength of each facility on each theme on a 4-point scale. We will then compare each theme across the higher and lower performing groups to identify the factors that differentiate higher versus lower performing facilities.

\section{DISCUSSION}

Scalable solutions to improve surgical quality in low-resource settings are urgently needed. This study will assess the effectiveness of the Safe Surgery 2020 multicomponent intervention as an approach to improving surgical quality. However, there are a number of risks to its successful implementation.

Measuring the impact of interventions to improve surgical quality in LMICs is challenging. Measures of surgical quality in low-resource settings are few, and measurement is difficult and expensive because of the lack of well-developed information systems. ${ }^{49}$ To generate high-quality evidence on the impact, we have incorporated several study design elements. First, the study uses mixed methods, which can provide a more comprehensive understanding of the impact and is important when evaluating a multicomponent intervention in a complex environment. ${ }^{475051}$ Second, the prospective study design and data collection through direct observation will allow 
for the collection of high-quality primary data, avoiding the pitfalls of retrospective chart review and reliance on secondary data. As echoed by Forrester et al direct observation is a more effective postoperative surveillance method than chart review. ${ }^{52}$ Third, baseline and endline data will be collected over 3 months to reduce the impact of monthly variation. ${ }^{53}$ Fourth, most studies evaluating surgical quality in East Africa are focused on a single tertiary hospital ${ }^{23} 275455$; this multicentred study will include facilities at the community, district and regional level and therefore improve the study's generalisability. The anticipated volume will also ensure that the study is adequately powered. Fifth, the quasi-experimental study design requires a focus on not just internal but also external validity, including factors related to intervention uptake such as acceptability to surgical providers, gathered through qualitative interviews, which is important for real-world implementation. ${ }^{56}$

To collect high-quality data, study will include several quality assurance mechanisms. First, we will pretest our tools in the local context until we are certain all identifiable barriers to successful data collection have been removed. Second, we will recruit and train Tanzanian medical data collectors, as they have the advantage of understanding the local context. Third, data will be collected through direct observation. Finally, weekly data quality checks will provide real-time feedback to the data collectors and provide additional quality control.

The study measures both processes and outcomes. Process measures can help to identify gaps and are actionable through QI activities. ${ }^{435}$ Systematic reviews on quality of essential surgical care in LMICs have shown that only $11 \%$ of published studies reported process measures. ${ }^{3}$ While patient outcomes are the ultimate measures of impact, they may not change in the short term and are difficult to reliably measure, especially in LMICs. A combination of process and outcome measures allows for a more multifaceted view of impact. Since the seminal study by Haynes et al, there has been mounting evidence on implementation of the Surgical Safety Checklist in improving morbidity and mortality. ${ }^{2} 13143057$ Evidence validating surgical quality indicators by directly linking them to patient outcomes is sparse. ${ }^{49}$ It is, therefore, important to build on the existing evidence ${ }^{30}$ to develop reliable metrics with consistent definitions that can be operationalised and effectively used to evaluate surgical quality in LMICs. Furthermore, it will be important to adapt the Surgical Safety Checklist to fit the Tanzanian context. Research suggests that adapting cognitive aids to the local context and pilot testing their fit are critical factors in successful implementation. ${ }^{58}$ Adaptation empowers local stakeholders to gain ownership of implementation success and can prevent checklist fatigue. ${ }^{59}$

Due to limited resources, the evaluation will not capture the development of SSI, sepsis and maternal sepsis cases postdischarge. A study in a rural hospital in Kenya has demonstrated that $72.9 \%$ of SSIs were diagnosed after discharge ${ }^{60}$ and postdischarge SSI rates in Ethiopian and Tanzanian hospitals were 21\%. ${ }^{61}{ }^{62}$ However, postdischarge diagnosis mechanisms such as phone surveillance have shown to be of low to moderate sensitivity in Tanzania and Kenya since they require patients to be able to reliably recognise the clinical features of wound infection, which can be difficult. ${ }^{5455}$

Cluster randomisation could reduce bias and confounding. However, we purposely selected control and intervention facilities for their geographic separation, to minimise the likelihood of exposure to the trainings in control regions. In addition, we selected intervention facilities with the highest surgical volume to ensure adequate power. However, we selected control and intervention facilities based on similar surgical volume, type of surgical cases (number of C-sections and general surgeries) and facility level to allow for a fair comparison between intervention and control sites.

Improvements in Surgical Safety Checklist use might be subject to the Hawthorne effect. However, data collectors' presence at the facilities for an extended period may allow them to build a relationship with the surgical teams, thus minimising the effect. ${ }^{63}$ Furthermore, the Checklist Observation Tool evaluates discussion of adherence to certain process measures as opposed to confirming their true implementation.

Finally, the study will measure the overall impact of the Safe Surgery 2020 intervention on surgical process and outcome measures. We will not be able to attribute changes to a particular intervention component.

\section{Ethics and dissemination}

Participation in the study is voluntary, and no monetary incentives will be provided to individual study participants. Verbal informed consent will be documented and obtained from all participants in Swahili. Parental consent will be obtained for those under the age of 18 .

Results will be presented to Tanzanian policy-makers and all study sites. We will share the study results with the scientific community through publications and conference presentations.

This study will provide rigorous evidence to the global surgical community about the effectiveness of the Safe Surgery 2020 approach to improving surgical care quality. If successful, patients and other stakeholders could benefit from a quality improvement method that could improve surgical outcomes in Tanzania's Lake Zone region and other regions with similar contexts.

\section{Author affiliations}

${ }^{1}$ Program in Global Surgery and Social Change, Harvard Medical School, Boston, Massachusetts, United States

${ }^{2}$ Department of Plastic and Oral Surgery, Boston Children's Hospital, Boston, MA, United States

${ }^{3}$ GE Foundation, Boston, Massachusetts, United States

${ }^{4}$ Safe Surgery 2020 Project, JHPIEGO, Dar es Salaam, Tanzania

${ }^{5}$ Department of Health, Social Welfare and Nutritional Service, President's Office Regional Administration and Local Government, Dodoma, Tanzania

${ }^{6}$ D-Implement, Dalberg Advisors, Nairobi, Kenya

${ }^{7}$ Assist International, Ripon, California, United States 
${ }^{8}$ Departments of Anesthesiology and Surgery, Boston Childrens Hospital, Boston, Massachusetts, United States

${ }^{9}$ The Innovations Unit, JHPIEGO, Baltimore, Maryland, United States

${ }^{10}$ Ministry of Health, Community Development, Gender, Elderly and Children, Dodoma, Tanzania

${ }^{11}$ Department of Adult Non-Communicable Diseases, Ministry of Health, Community Development, Gender, Elderly and Children, Dodoma, Tanzania

Acknowledgements We would like to acknowledge and thank all organisations and individuals who contributed to our early consultation on the design of the Safe Surgery 2020 multicomponent intervention as well as those organisations and individuals who have contributed to the larger Safe Surgery 2020 initiative including Assist International, Dalberg, Jhpiego, LifeBox, Project ECHO, SPECT and WFSA. We would like to express our sincere appreciation to the Ministry of Health, Community Development, Gender, Elderly and Children (MoHCDGEC) President's OfficeRegional Administration and Local Government in Tanzania (PO-RALG), the regional medical officers of Mara, Kagera, Shinyanga, Simiyu and Geita regions and the frontline leadership and surgical team members in the study sites for their support of the study. We would like to thank Amanda DiMeo, Desmond Jumbam, William Lodge II, Meaghan Sydlowski and for their important contributions to the field procedures. We would like to thank Christina Smiraglia for her editing assistance.

Contributors JM, SA, SK, GM, IC, SJS and DZ designed the study methods. SA, SK, GM, TNL and SJS drafted the manuscript. DB, AH, NAK, EM, CR, AT, AV, MU and SM were involved in the conception and design of the programme and evaluation and critically reviewed and approved the final manuscript.

Funding This project was funded under grant number 5R18HS024235-02 from the GE Foundation and grant number 17-F0012 from ELMA Philanthropies.

Disclaimer The funders will not have any input on data collection, analysis or interpretation of the data. Further, they will not have any authority over the writing of reports or the decision to submit findings for publication.

Competing interests All authors have completed the ICMJE uniform disclosure form at www.icmje.org/coi_disclosure.pdf and declare: SA, SK, GM, IC, JM, TNL, AH, EM, AT and CR had financial support from GE Foundation for the submitted work; AT and AH declare financial support from ELMA Philanthropies; IC declares payments in kind (flights/hotel) from the Rhonda Stryker Foundation for work in the field of global surgery, but not directly for this manuscript or related work; and AV and DB are employed by GE Foundation, which funded this work. The authors have had no financial relationships with any organisations that might have an interest in the submitted work in the previous 3 years and no other relationships or activities that could appear to have influenced the submitted work.

\section{Patient consent for publication Not required.}

Ethics approval We obtained ethical approval from both Harvard Medical School and Tanzania's National Institute for Medical Research.

Provenance and peer review Not commissioned; externally peer reviewed.

Open access This is an open access article distributed in accordance with the Creative Commons Attribution Non Commercial (CC BY-NC 4.0) license, which permits others to distribute, remix, adapt, build upon this work non-commercially, and license their derivative works on different terms, provided the original work is properly cited, appropriate credit is given, any changes made indicated, and the use is non-commercial. See: http://creativecommons.org/licenses/by-nc/4.0/.

ORCID iD

Shehnaz Alidina http://orcid.org/0000-0002-4403-7871

\section{REFERENCES}

1. Meara JG, Leather AJM, Hagander L, et al. Global surgery 2030: evidence and solutions for achieving health, welfare, and economic development. The Lancet 2015;386:569-624.

2. Haynes AB, Weiser TG, Berry WR, et al. A surgical safety checklist to reduce morbidity and mortality in a global population. $N$ Engl J Med 2009:360:491-9.

3. Saluja S, Mukhopadhyay S, Amundson JR, et al. Quality of essential surgical care in low- and middle-income countries: a systematic review of the literature. Int J Qual Health Care 2019;31:166-72.

4. Kruk ME, Gage AD, Arsenault C, et al. High-Quality health systems in the sustainable development goals era: time for a revolution. The Lancet Global Health 2018;6:e1196-252.
5. Rowe AK, Rowe SY, Peters DH, et al. Effectiveness of strategies to improve health-care provider practices in low-income and middle-income countries: a systematic review. Lancet Glob Health 2018:6:e1163-75.

6. Grimshaw JM, Thomas RE, MacLennan G, et al. Effectiveness and efficiency of guideline dissemination and implementation strategies. Int J Technol Assess Health Care 2005;21:149.

7. Leslie $\mathrm{HH}$, Gage A, Nsona $\mathrm{H}$, et al. Training and supervision did not meaningfully improve quality of care for pregnant women or sick children in sub-Saharan Africa. Health Aff 2016;35:1716-24.

8. Squires JE, Sullivan K, Eccles MP, et al. Are multifaceted interventions more effective than single-component interventions in changing health-care professionals' behaviours? an overview of systematic reviews. Implementation Sci 2014;9.

9. Wensing M, van der Weijden T, Grol R. Implementing guidelines and innovations in general practice: which interventions are effective? $\mathrm{Br}$ J Gen Pract 1998;48:991-7.

10. Epiu I, Tindimwebwa JVB, Mijumbi C, et al. Working towards safer surgery in Africa; a survey of utilization of the who safe surgical checklist at the main referral hospitals in East Africa. BMC Anesthesiol 2015;16:60.

11. Borchard A, Schwappach DLB, Barbir A, et al. A systematic review of the effectiveness, compliance, and critical factors for implementation of safety checklists in surgery. Ann Surg 2012;256:925-33.

12. Treadwell JR, Lucas S, Tsou AY. Surgical checklists: a systematic review of impacts and implementation. BMJ Qual Saf 2014;23:299-318.

13. Kwok AC, Funk LM, Baltaga R, et al. Implementation of the world Health organization surgical safety checklist, including introduction of pulse oximetry, in a resource-limited setting. Ann Surg 2013;257:633-9.

14. de Vries EN, Prins HA, Crolla RMPH, et al. Effect of a comprehensive surgical safety system on patient outcomes. N Engl J Med 2010;363:1928-37.

15. Papaconstantinou HT, Jo C, Reznik SI, et al. Implementation of a surgical safety checklist: impact on surgical team perspectives. Ochsner J 2013;13:299-309.

16. Molina G, Jiang W, Edmondson L, et al. Implementation of the surgical safety checklist in South Carolina hospitals is associated with improvement in perceived perioperative safety. J Am Coll Surg 2016;222:725-36.

17. Böhmer AB, Wappler F, Tinschmann T, et al. The implementation of a perioperative checklist increases patients' perioperative safety and staff satisfaction: Patients' perioperative safety and staff satisfaction. Acta Anaesthesiol Scand 2012;56:332-8.

18. Singer SJ, Molina G, Li Z, et al. Relationship between operating room teamwork, contextual factors, and safety checklist performance. $J$ Am Coll Surg 2016;223:568-80.

19. Eriksson J, Baker T, Jörnvall H, et al. Quality of anaesthesia for caesarean sections: a cross-sectional study of a university hospital in a low-income country. Trop Med Int Health 2015;20:1329-36.

20. Nelissen EJT, Mduma E, Ersdal HL, et al. Maternal near miss and mortality in a rural referral hospital in northern Tanzania: a crosssectional study. BMC Pregnancy Childbirth 2013;13:141.

21. Maegga BTA, Cox J, Malley KD. Malaria in the southern highlands of Tanzania: a review of hospital records. Tanzan Health Res Bull 2005;7:125-32.

22. Mpogoro FJ, Mshana SE, Mirambo MM, et al. Incidence and predictors of surgical site infections following caesarean sections at Bugando medical centre, Mwanza, Tanzania. Antimicrob Resist Infect Control 2014;3:25.

23. Eriksen HM, Chugulu S, Kondo S, et al. Surgical-Site infections at Kilimanjaro Christian medical center. J Hosp Infect 2003;55:14-20.

24. Rothe C, Schlaich C, Thompson S. Healthcare-Associated infections in sub-Saharan Africa. J Hosp Infect 2013;85:257-67.

25. Anderson DJ. Surgical site infections. Infect Dis Clin North Am 2011;25:135-53.

26. Public Health England. Surveillance of surgical site infections in NHS hospitals in England: 2014 to 2015 2015:1-44.

27. De Nardo P, Gentilotti E, Nguhuni B, et al. Post-caesarean section surgical site infections at a Tanzanian tertiary Hospital: a prospective observational study. J Hosp Infect 2016;93:355-9.

28. Ward VP, Charlett A, Fagan J, et al. Enhanced surgical site infection surveillance following caesarean section: experience of a multicentre collaborative post-discharge system. J Hosp Infect 2008;70:166-73.

29. Rauk PN. Educational intervention, revised instrument sterilization methods, and comprehensive preoperative skin preparation protocol reduce cesarean section surgical site infections. Am J Infect Control 2010;38:319-23.

30. Forrester JA, Koritsanszky LA, Amenu D, et al. Developing process maps as a tool for a surgical infection prevention quality 
improvement initiative in resource-constrained settings. J Am Coll Surg 2018;226:1103-16.

31. World Bank. Poverty \& Equity Brief: Tanzania, 2018. Available: http:// databank.worldbank.org/data/download/poverty/33EF03BB-97224AE2-ABC7-AA2972D68AFE/Global_POVEQ_MDG.pdf [Accessed 15 Feb 2019].

32. Statement on maternal sepsis. World Health organization, 2017. Available: https://apps.who.int/iris/bitstream/handle/10665/254608/ WHO-RHR-17.02-eng.pdf? sequence $=1$

33. Langabeer JR, DelliFraine JL, Heineke J, et al. Implementation of lean and six sigma quality initiatives in hospitals: a goal theoretic perspective. Oper Manag Res 2009;2:13-27.

34. Frankel HL, Crede WB, Topal JE, et al. Use of corporate six sigma Performance-Improvement strategies to reduce incidence of catheter-related bloodstream infections in a surgical ICU. J Am Coll Surg 2005;201:349-58.

35. Birkmeyer JD, Dimick JB, Birkmeyer NJO. Measuring the quality of surgical care: structure, process, or outcomes? J Am Coll Surg 2004;198:626-32.

36. Zhou C, Crawford A, Serhal E, et al. The impact of Project echo on participant and patient outcomes. Academic Medicine 2016;91:1439-61.

37. National surgical, obstetric and anaesthesia plan (NSOAP), 2018

38. Harris PA, Taylor R, Thielke R, et al. Research electronic data capture (REDCap)-A metadata-driven methodology and workflow process for providing translational research informatics support. J Biomed Inform 2009;42:377-81.

39. Huang LC, Conley D, Lipsitz S, et al. The surgical safety checklist and teamwork coaching tools: a study of inter-rater reliability. BMJ Qual Saf 2014;23:639-50.

40. Mangram AJ, Horan TC, Pearson ML, et al. Guideline for prevention of surgical site infection, 1999. Hospital infection control practices Advisory Committee. Infect Control Hosp Epidemiol 1999;20:250-78.

41. Comstedt P, Storgaard M, Lassen AT. The systemic inflammatory response syndrome (SIRS) in acutely hospitalised medical patients: a cohort study. Scand J Trauma Resusc Emerg Med 2009;17:67.

42. Bone RC, Balk RA, Cerra FB, et al. Definitions for sepsis and organ failure and guidelines for the use of innovative therapies in sepsis. The ACCP/SCCM consensus conference Committee. American College of chest Physicians/Society of critical care medicine. Chest 1992;101:1644-55.

43. Surviving Sepsis Campaign. Evaluation for severe sepsis screening tool, 2012. Available: http://www.survivingsepsis.org/ [Accessed 15 Feb 2016].

44. Surviving Sepsis Campaign. Maternal assessment of sepsis, 2013. Available: http://www.survivingsepsis.org/ [Accessed 15 Feb 2019].

45. Medical records manual: a guide for developing countries. Geneva: : World Health Organization 2002.

46. Singer SJ, Hayes J, Cooper JB, et al. A case for safety leadership team training of hospital managers. Health Care Manage Rev 2011;36:188-200.

47. Bradley EH, Curry LA, Taylor LA, et al. A model for scale up of family health innovations in low-income and middle-income settings: a mixed methods study. BMJ Open 2012;2:e000987.

48. Glaser BG. The constant comparative method of qualitative analysis. Soc Probl 1965;12:436-45.

49. Citron I, Saluja S, Amundson J, et al. Surgical quality indicators in low-resource settings: a new evidence-based tool. Surgery 2018;164:946-52.
50. Fetters MD, Curry LA, Creswell JW. Achieving integration in mixed methods Designs-Principles and practices. Health Serv Res 2013;48:2134-56.

51. Creswell JW, Plano Clark VL. Designing and conducting mixed methods research. Thousand Oaks, Calif: SAGE Publications, 2007.

52. Forrester JA, Koritsanszky L, Parsons BD, et al. Development of a surgical infection surveillance program at a tertiary hospital in Ethiopia: lessons learned from two surveillance strategies. Surg Infect 2018;19:25-32.

53. Biccard BM, Madiba TE, Kluyts $\mathrm{H}-\mathrm{L}$, et al. Perioperative patient outcomes in the African surgical outcomes study: a 7-day prospective observational cohort study. The Lancet 2018;391:1589-98.

54. Aiken AM, Wanyoro AK, Mwangi J, et al. Evaluation of surveillance for surgical site infections in Thika Hospital, Kenya. J Hosp Infect 2013;83:140-5.

55. Nguhuni B, De Nardo P, Gentilotti E, et al. Reliability and validity of using telephone calls for post-discharge surveillance of surgical site infection following caesarean section at a tertiary hospital in Tanzania. Antimicrob Resist Infect Control 2017;6.

56. Handley MA, Lyles CR, McCulloch C, et al. Selecting and improving quasi-experimental designs in effectiveness and implementation research. Annu Rev Public Health 2018;39:5-25.

57. Singer SJ, Jiang W, Huang LC, et al. Surgical team member assessment of the safety of surgery practice in 38 South Carolina hospitals. Med Care Res Rev 2015;72:298-323.

58. Alidina S, Goldhaber-Fiebert SN, Hannenberg AA, et al. Factors associated with the use of cognitive AIDS in operating room crises: a cross-sectional study of US hospitals and ambulatory surgical centers. Implementation Sci 2018;13.

59. Ariadne Labs. Safe surgery checklist implementation guide. Boston,MA: Ariadne Labs, 2015. www.SafeSurgery2015.org

60. Nthumba PM, Stepita-Poenaru E, Poenaru D, et al. ClusterRandomized, crossover trial of the efficacy of plain soap and water versus alcohol-based rub for surgical hand preparation in a rural hospital in Kenya. Br J Surg 2010;97:1621-8.

61. Amenu D, Belachew T, Araya F. Surgical site infection rate and risk factors among obstetric cases of jimma university specialized Hospital, Southwest Ethiopia. Ethiop J Health Sci 2011;21:91-100.

62. Fehr J, Hatz C, Soka I, et al. Risk factors for surgical site infection in a Tanzanian district Hospital: a challenge for the traditional national nosocomial infections surveillance system index. Infect Control Hosp Epidemiol 2006;27:1401-4.

63. Sedgwick P, Greenwood N. Understanding the Hawthorne effect. BMJ 2015;351.

64. Darouiche RO, Wall MJ, Itani KMF, et al. Chlorhexidine-Alcohol versus povidone-iodine for surgical-site antisepsis. N Engl J Med 2010;362:18-26.

65. Haas DM, Morgan S, Contreras K, et al. Vaginal preparation with antiseptic solution before cesarean section for preventing postoperative infections. Cochrane Database Syst Rev 2018;30.

66. Wilms MC, Mbembela O, Prytherch $\mathrm{H}$, et al. An in-depth, exploratory assessment of the implementation of the National health information system at a district level hospital in Tanzania. BMC Health Serv Res 2014;14.

67. Nyamtema AS. Bridging the gaps in the health management information system in the context of a changing health sector. $B M C$ Med Inform Decis Mak 2010;10. 